\title{
The Boundary Condition's Study and Application of Diesel Dynamics Analysis on Unsteady Working Condition
}

\author{
HUANG Ying-yun \\ Power Engineering College \\ Naval University of Engineering \\ Wuhan, China
}

\author{
GAO Hao-peng \\ Power Engineering College \\ Naval University of Engineering \\ Wuhan, China \\ gaohaopeng@126.com
}

\author{
SUN Yu-peng \\ Power Engineering College \\ Naval University of Engineering \\ Wuhan, China
}

\begin{abstract}
With the instruction of computational dynamics of multi-body systems theory, the system of rigid bodies and rigidflexible bodies model of the diesel are built using CAE technology. Based on the models, considering the factors of fluid-solid coupling, modal coupling and feedback control technology, the rational boundary condition of diesel's unsteady working condition is analyzed; it contains: cylinder pressure, transient speed, constraint relation and the simulation of the main bearing. The dynamics strength is analyzed using the models on unsteady working condition. The dynamics stress distributing is gotten in different working condition. The modeling and analyzing process can apply to the optimum design of diesel structure, the study of matching of ship-engine-oar and other marine machinery.
\end{abstract}

Keywords-boundary condition; computational dynamics of multi-body systems; diesel; rod

\section{INTRODUCTION (HEADING 1)}

The strength analysis of power transfer subassembly is a necessary procedure in security check of diesel. With the development of computer hardware and applied technology, the dynamic strength analysis of these complicated machine is feasible ${ }^{[1]}$. At the present time, the strength analysis method of diesel mainly combines the computational dynamics of multibody systems method and finite element method ${ }^{[2]}$.

Although the simulation reduces the calculating by hand, the model's correctness and the boundary condition's rationality are needed more strictly. The boundary conditions of diesel's dynamics analysis mainly contains: the constraint relation of Subassembly, the firing order, the reverse torque, cylinder pressure, transient speed, and the simulation of the main bearing. At present, most studies are limited to argument steady working condition, but few focus on the unsteady working condition. Based on this conclusion, the result of strength analysis in this paper is more accurate.

\section{THE FOUNDATION OF MULTI-BODY SYSTEM MODEL}

A. The foundation of system of rigid bodies dynamics model

The study in this paper bases on the computational dynamics of multi-body systems method. The foundation of 3D model is the basement of system simulation, and whether the model is built correctly or not directly affect the correctness of the result. The movement of power transfer subassembly of diesel is complex, and there is coupling effect among the subassembly during motion, so the whole model of the type is built using Pro/E software. Then, the system of rigid bodies dynamics model is built using the interface of data transfer which can link Pro/E software and computational dynamics of multi-body systems software (as shown in Figure 1).

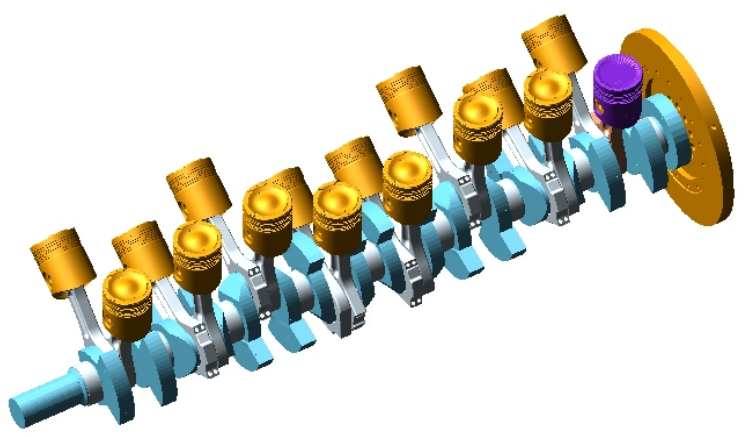

Figure 1. System of rigid bodies model of diesel

\section{B. The foundation of the system of rigid-flexible bodies model}

Based on the foundation of system of rigid bodies dynamics model, the crankshaft, connecting rod and piston are flexible, and the MNF files are gained. Then the rigid-flexible model is formed using the method which can replace the rigid bodies as flexible bodies. The process relies on the MNF files ${ }^{[3]}$. In the course of MNF generation, the addition of MPC is necessary. The purpose is to actualize the feasible transfer of force and motion. The substance of MPC addition is to build the link of movement, and the addition substantially changes the structure of flexible bodies. But the change chimes in with the constraint relation of rigid bodies during dynamics analysis of diesel, so it is logical. In the course of flexible bodies generation, considering the calculational speediness and resultant reliability, the hexahedron grids of the subassembly are formed (as shown in Figure 2). 

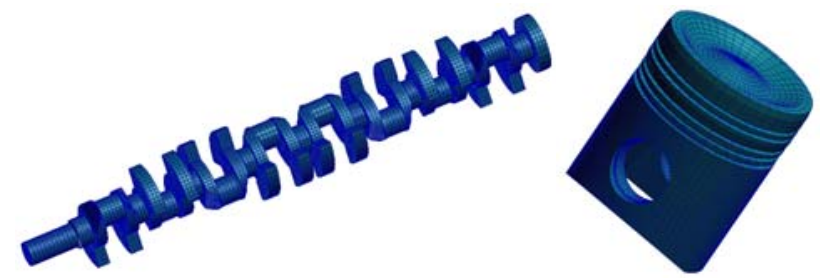

Figure 2. The hexahedron grids of the subassembly

\section{THE STUDY ON BOUNDARY CONDITION}

As a kind of heat energy machine, the diesel has many systems, so the boundary conditions are comparatively complex. The primary study objects of boundary conditions in this paper are power transfer subassembly. Whether the boundary condition is added correctly or not, it directly affects the correctness of the model and the correctness of the solutions. In this paper, according the computational dynamics of multi-body systems theory and the characteristic of diesel, the boundary conditions of dynamics analysis mainly contain: the constraint relation of Subassembly, the firing order and firing delivery phase, the reverse torque, cylinder pressure, transient speed, and the simulation of the main bearing.

\section{A. The analysis of constraint relation}

Telling simply form the process of modeling and resolving, the relationship is to build the different equation set of rigid body and rigid body, rigid body and flexible body, flexible body and flexible body. So the constraint relation directly affects the correctness of the solutions. The mode of constraint relation should base on the movement connection of actual member. Then the form of degrees of freedom is abstracted, and the types of constraints are gained. The constraint relation in this paper is as shown in Figure $3^{[4]}$.

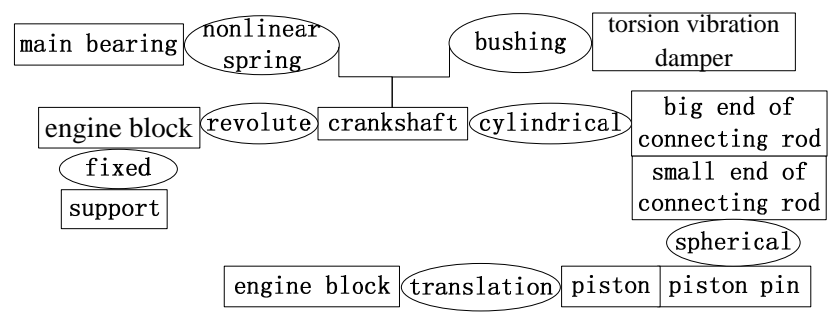

Figure 3. The constraint relation of multi-body system

\section{B. The additions of cylinder pressure and outside force}

The structure of the diesel is $16 \mathrm{~V}$. The firing order of the diesel is regular for the following types: A1-B4-A5-B2-A7-B6A3-B1-A8-B5-A4-B7-A2-B3-A6-B8-A1; the firing delivery phase angle is $30^{\circ}$ or $60^{\circ}$. At the diesel's work condition, there is a balance between the torque generated by cylinder pressure and the stalling torque generated by load, so the outside forces in this paper refer chiefly to cylinder pressure and stalling torque. The data of cylinder pressure is measured on the diesel's rated condition. The primary principle of measurement is to use the pulse signal generated by count circuit of encoder to drive the acquisition circuits. The NI system acquire data when the crankshaft turn every degree (as shown in Figure 4).
At the dynamics analysis, the pressure data of whole process is gained using interpolation method, which bases on the measurement data.

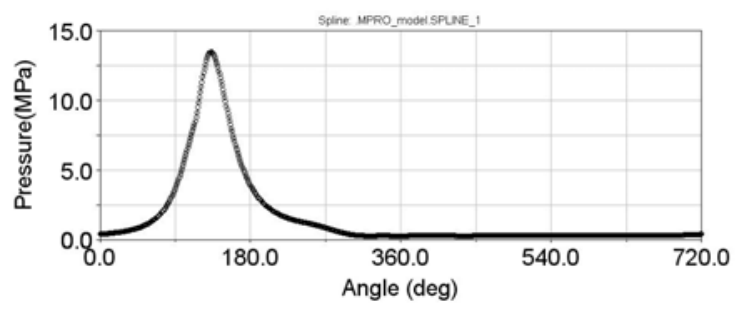

Figure 4. The measure curve of cylinder pressure

The unsteady working condition refers chiefly to the speedup and speed-down working condition. On the speeddown working condition, the strength of diesel has a big margin, so the authors pay more attention to speedup working condition. It contains emergent and natural speedup when the diesel is at warm-up state or unwarmed state ${ }^{[5]}$. In addition, the strength is analyzed on circumgyratetion condition of full rudder.

The study of strength analysis of diesel is mainly localized in the rated state ${ }^{[6]}$, so there is few study on the addition of boundary conditions about unsteady working condition. Because of the long time of the speedup process, it is difficult to acquire data through the whole course. A periodical data of cylinder pressure is acquired on the speedup condition when the rotate speed increases every $100 \mathrm{r} / \mathrm{min}$. Based on these data, the whole data of unsteady condition is gained using the program to solve, which is compiled by SIMULINK software. Then the data in the software of the computational dynamics of multi-body systems is added by interpolation and data fitting method. The curve in the Figure 5 is the data of cylinder pressure when the diesel works at the circumgyratetion condition of full rudder. It is feasible to measure the power at the unsteady working points, so the stalling torque can be calculated by the equation ( $M_{e}=9550 * N_{e} / n$ ) between power and torque. The movement state of diesel is determined by interaction result of cylinder pressure and stalling torque.

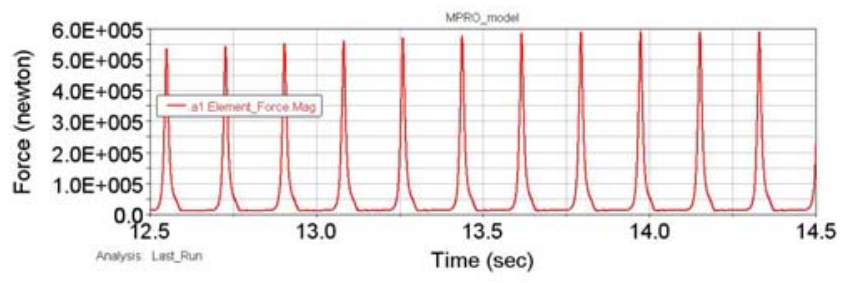

Figure 5. The curve of cylinder pressure in circumgyratetion condition of full rudder

\section{The analysis of transient speed}

According to the principle of diesel's governor, the number of cylinder pressure should be multiplied by the coefficient to make the balance between all the cylinder's torque and reverse torque. The coefficient comes from the step function, and the function is $\operatorname{step}((\mathrm{n}-\mathrm{N}),-\mathrm{a}, \mathrm{b}, \mathrm{a}, \mathrm{c})$; in the function, the $\mathrm{n}$ stands for , the $\mathrm{N}$ stands for , the parameters of $\mathrm{a}, \mathrm{b}$ and $\mathrm{c}$ are gained by 
simulation experimentation. This method can implement the adding of transient speed at transient working condition, and the speed curve in start-up working condition is as shown in the Figure 6. The number of start-time is controlled by the coefficient of reverse torque.

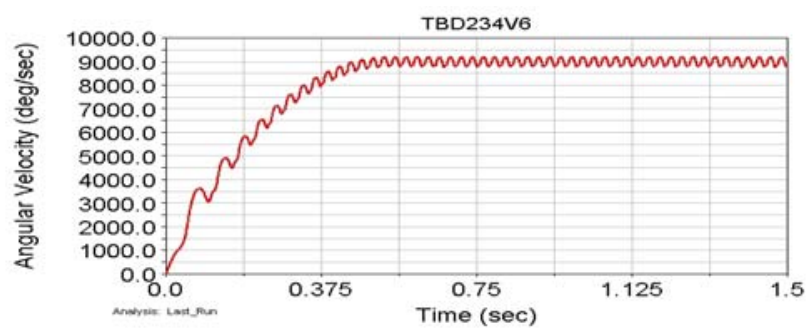

Figure 6. The change of rotate speed in start-up working condition

\section{The simulation of main bearing}

The simulation of main bearing is extremely important to the dynamics analysis on crank system. When the diesel is working, because of the constraint of the main bearing, it affects the axial and radial movement of crank. Used hydrodynamic lubrication, the oil film of main bearing plays a very important role, and it contains: supporting crank, bearing the load of cylinder pressure and reducing the vibration of crank. The simulation types of main bearing mainly contain: linear spring, nonlinear spring and hydro-FEM; and at present, most studies are limited to static state simulation. This paper considers the factors of the oil film dynamic stiffness and damping while simulating the component's rotary movement; then using the method of calculator program and feedback control technology makes the fluid-solid coupling possible.

The calculated equations of oil film stiffness and damping ${ }^{[7]}$ are as shown in formula (1) and formula (2). In the formulas, the $\psi$ stands for clearance ratio, the $\mu$ stands for oil viscosity, the $L$ stands for bearing's width, the $\omega$ stands for transient angle speed, and the W stands for dimensionless outside load. This paper makes the transient speed parameterized, at the same time measures the transient speed continuously; and the data are calculated and exchanged by MATLAB; then the stiffness and damping are obtained. Based on this, using nonlinear spring simulates the main bearing, and the sketch map is as shown in Figure 7.

$$
\begin{gathered}
K_{i j}=k_{i j} \frac{\psi^{3}}{\mu \omega L W} \quad(\mathrm{i}, \mathrm{j}=\mathrm{y}, \mathrm{z}) \\
C_{i j}=c_{i j} \frac{\psi^{3}}{\mu L W} \quad(\mathrm{i}, \mathrm{j}=\mathrm{y}, \mathrm{z})
\end{gathered}
$$

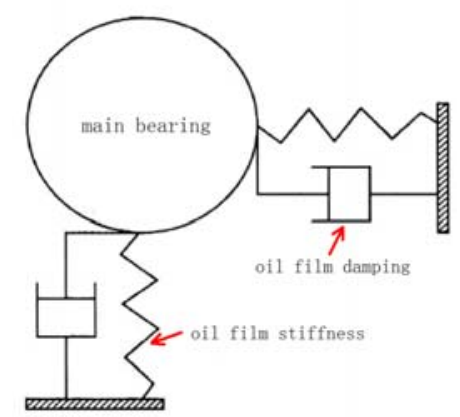

Figure 7. The sketch map of head-axletree sustentation

\section{THE DYNAMIC STRENGTH ANALYSIS ON VARYING DUTY}

The strength is checked on the rated working condition when the diesel is designed and made; but on varying duty the dynamic distribution coefficient can reduce the reliability of strength; so it is necessary to combine the load characteristics curve to study the dynamic strength on unsteady working condition.

Based on the analysis of boundary condition on unsteady working condition, this paper mainly analyses the strength of unsteady condition in common use. The results calculated in different conditions are compared and analyzed (take the rod as example). The Figure 8 is the stress nephogram of crankshaft in rated working condition. Form the Figure 8, it can be found that the max value of stress is $157.5 \mathrm{MPa}$ (the limit number of yielding is $930 \mathrm{MPa}$ ). The max value of stress of crankshaft is respectively 183.2MPa, $175.7 \mathrm{MPa}, 174.2 \mathrm{MPa}, 166.0 \mathrm{MPa}$ and 131.7MPa; the corresponding working conditions are emergent speedup at warm-up state, emergent speedup at unwarmed state, natural speedup at warm-up state, natural speedup at unwarmed state, and circumgyratetion condition of full rudder. The Figure 9 is the stress curve of rod at emergent speedup at warm-up state, and the Figure 10 is the stress curve of rod at circumgyratetion condition of full rudder. It can come to some conclusions by analyzing the data, chart and animation. Firstly, among the working conditions the max stress of crankshaft is at the emergent speedup at warm-up state. The value is 1.17 times as big as that at rated state. The reasons are that the time of speedup is shorter and the change of power is larger. Secondly, under the same condition of cylinder pressure the value of max stress at the high speed is larger than that at the low speed. It indicates that the inertial force of power transfer subassembly has significant influence on the dynamic strength. Thirdly, the amplitude of stress at emergent speedup at unwarmed state is smaller than that at emergent speedup at warm-up state, but we should pay more attention to the emergent speedup at unwarmed state. Because the oil film pressure at main bearing and piston ring have not been built when the diesel is at unwarmed state. Fourthly, the diesel works at the restrained line of power, but the absolute value of power and speed is little at the circumgyratetion condition of full rudder. So the amplitude of stress is not so big. 


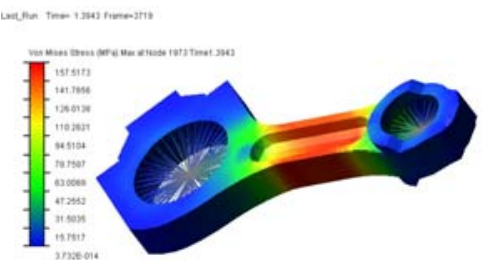

Figure 8. The stress nephogram of rod in rated condition

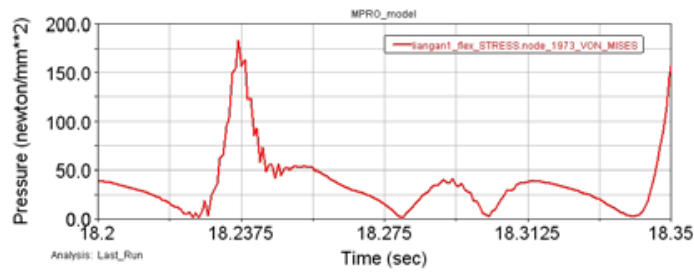

Figure 9. The stress curve of rod in an emergent speedup at diesel's warm-up state

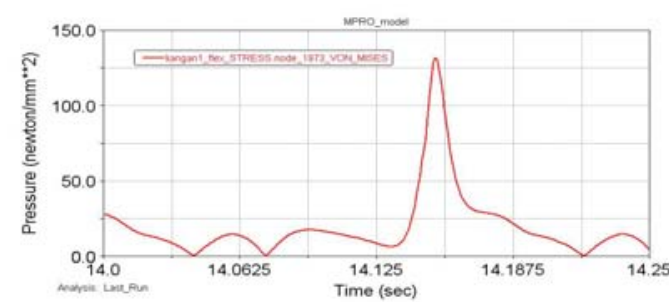

Figure 10. The stress curve of rod in circumgyratetion condition of full rudder

\section{CONCLUSION}

At present, the method of strength analysis on the rated working condition is popular and efficient; but at the operation management, it indicates by many accidents that the reliability of strength decreases a little; so it is more significant to study the strength of diesel on the unsteady working condition.

Certainly, the dynamic strength analysis at unsteady state is complex than that at rated state. The development of computer hardware and computer application technology makes the dynamic strength analysis possible. The authors combine the computational dynamics of multi-body systems method and finite element method in this paper, and analyze the modeling and resolving of computational dynamics of multi-body systems, especially analyze the boundary conditions on unsteady state by the numbers. The results show that the amplitude of stress is big on speedup condition. There are many factors that we must pay attention to at unsteady state, such as the large change of power, the little stability of governor, the biggish acceleration and inertial force of power transfer subassembly, and many non-linear conditions. The further research work should be done about dynamic strength analysis on unsteady working condition.

\section{ACKNOWLEDGMENT}

The research described in this paper is supported by the natural science foundation of Naval University of Engineering. The authors wish to express their thanks to Prof. Ouyang Guangyao and his team for their permission and active support during the engine testing program.

\section{REFERENCES}

[1] Hao Zhi-yong, Duan Xiu-bing, Cheng Jin-lin, "Flexible Crankshaft System of A Vehicle Diesel Engine,” RAILWAY LOCOMOTIVE \& CAR, vol. 23, Nov. 2003, pp. 86-89.

[2] Wu Nan, Liao Ri-dong, Zhang Bao-cheng, "Multi-Body System Dynamics Analysis of the Crank and Connecting Rod Mechanism in Diesel Engines,” Chinese Internal Combustion Engin Engineering, vol. 26, Oct. 2005, pp. 69-73.

[3] Zhang Guo-qing, Huang Bo-chao, and Pu Geng-qiang, "Dynamical Simulation of Crankshaft System of Automobile Engine,” Journal of System Simulation, vol. 18, Aug 2006, pp. 2293-2295.

[4] Gao Hao-peng, Huang Ying-yun, and Wang Xue-shan, "Study on methods of dynamic analysis on moving mechanism of marine diesel," Journal of Vibration and Shock, vol. 28, Aug 2009, pp. 84-87.

[5] Wang Yong-sheng. Research on steady-state and dynamic characteristics and operation scheme of combined diesel and diesel propulsion plant. Wuhan: Hua-zhong university of science\&technology, 2005.

[6] Sun Jun, Gui Chang-lin, and Li Zhen, “A review of crankshaft strength analysis for internal combustion engines," Transactions of CSICE, vol. 20, Feb. 2002, pp. 179-184.

[7] Kong Jing-zhi, "Practicalm Method for Vibration Analysis on Internal Combustion Engine Crankshaft System,” Journal of Vibration and Shock, vol. 28, Nov. 2009, pp. 195-198. 